\title{
Development and Characterization of Atorvastatin Calcium Sustained Release Tablet Using Carbomer-974 and Hypromellose-15000 cps
}

\author{
S. Akbar, R. Bhatta, M. A. Rahman, M. S. Hossain*, M. S. Bhuiyan, M. G. Uddin \\ Department of Pharmacy, Noakhali Science and Technology University, Sonapur, Noakhali- 3814, \\ Bangladesh
}

Received 10 December 2016, accepted in final revised form 12 March 2017

\begin{abstract}
A new sustained release formulation of Atorvastatin Calcium tablet, exhibiting improved swelling property and compatibility to prolong the drug release was prepared. Sustained release tablets were formulated using varying concentration of Carbomer-974 and Hypromellose-15000 cps, by direct compression method. Physical characteristics, compactibility, swelling index and drug content uniformity of the prepared formulations were determined. The drug release studies was carried out in USP dissolution test apparatus II (paddle) using phosphate buffer of $\mathrm{pH} 6.8$ as dissolution medium for 8 hours and documented the effects of polymers on the drug release profile. The release mechanism was explored and explained with Zero order \& Higuchi kinetic models. Formulation F1 and F5 were best fitted in the Higuchi model, representing diffusion mechanism of drug release, while Formulations F2, F3 and F4 showed Zero order model of drug release profile. The drug release pattern, compactibility and swelling index property of the formulated preparations were concentration dependent of the polymers used. Further study is necessary to evaluate the in vitro-in vivo relationship, but this study will be helpful for future to exploit the potential of this drug delivery system for the benefit of the mankind.
\end{abstract}

Keywords: Atorvastatin Calcium; Sustained release; Carbomer-974; Hypromellose-15000 cps.

(C) 2017 JSR Publications. ISSN: 2070-0237 (Print); 2070-0245 (Online). All rights reserved. doi: http://dx.doi.org/10.3329/jsr.v9i2.30600 J. Sci. Res. 9 (2), 247-254 (2017)

\section{Introduction}

Oral route of drug delivery system is widely considered acceptable by the patients for the administration of therapeutically active drugs compared to other routes. Various oral sustained release delivery systems have been developed with the aim to achieve a prolonged therapeutic effect. Sustained release or controlled release delivery systems have been accounted for the predictable and reproducible drug release with enhanced duration of action, reduced toxicity and better patient compliance [1]. Generally, different techniques which are experimented to modify the release of drug from dosage forms are

*Corresponding author: pharmasalimres@gmail.com 
coated beads, granules and microspheres, multi tablet system, micro encapsulated drug, complex formation, ion exchange resins, and embedding drug in slowly eroding or hydrophilic matrix system. Among others documented methods for formulation of sustained release dosage form, the simplest and convenient technology is matrix system, where the drugs are embedded in polymer and excipients blends [2]. The oral drug delivery is the most widely utilized route of administration among all the routes for the treatment of most of the pathological conditions.

Recently, various disease conditions such as atherosclerosis, diabetes, obesity etc are rising up day by day due to the enhancement of lipid profile inside the body. Atorvastatin Calcium lowers the lipid profile, triglycerides and cholesterol, level of blood in the body and normally prescribed for the treatment of pathological conditions such as hyperlipidemias, atherosclerosis and other cardiovascular diseases related to lipid profile. Atorvastatin competitively inhibits the enzyme HMG-CoA reductase, which converts 3hydroxy-3-methylglutaryl coenzyme A to mevalonate, a precursor of sterols [3]. It inhibits sterol synthesis after single oral dose for approximately 1 to $8 \mathrm{~h}$. The bioavailability of Atorvastatin Calcium is $13 \%$ to $14 \%$ owing to a major hepatic first pass metabolism. Hence, it is important to evaluate drug features to properly utilize its effect, such as the stability, physical characteristics and compatibility of the pharmaceutical formulation, since any changes can directly influence its bioavailability and release profile.

Previously we reported the use of different polymers like methyl cellulose, Kollidon SR, HPMC for sustained release dosage form design [4,5] while other researchers mentioned the use of PVP-K30 and Locust bean gum as a rate retardant in floating drug delivery [6]. The present investigation was focused to develop Atorvastatin Calcium sustained release tablet to evaluate the effect of polymer Carbomer-974 and Hypromellose- $15000 \mathrm{cps}$ on drug release behavior from the prepared formulations.

\section{Experimental}

\subsection{Materials}

Atorvastatin Calcium was received as a gift sample from NIPRO JMI Pharma Ltd, Bangladesh. Polymer Carbomer-974 (MW 72.02 g/mol, purity > 90\%) and Hypromellose15000 cps (MW $86 \mathrm{Kd}$, purity > 90\%) were obtained as a gift sample from Eskayef Bangladesh Limited. Other materials were obtained as a gift samples from BASF chemical company, Bangladesh. All the samples were stored under appropriate conditions. Analytical reagent grade chemicals were used in this study and distilled water was used throughout the experiment.

\subsection{Preparation of sustained release tablet}

Atorvastatin Calcium sustained release tablets, each containing $20 \mathrm{mg}$ of Atorvastatin Calcium were prepared by direct compression technique. The composition of all formulations of this study is presented in Table 1. The active ingredient and other 
excipients were accurately weighed for forty tablets according to the formulations and were blended in a laboratory designed small drum blender. Particular attention has been given to ensure thorough mixing and phase homogenization. Individually weighed the amount of granules for each tablet $(325 \mathrm{mg}$ ) and compressed by using a Perkin-Elmer laboratory hydraulic press equipped with a $10 \mathrm{~mm}$ flat faced die and punch set. The surface of the die and punch were lubricated with magnesium stearate before compression of each tablet. Prepared tablets were stored at room temperature in airtight photo film container. The technique for tablet production was followed as mentioned previously [7-9].

Table 1. Formulation of Atorvastatin Calcium sustained release tablet.

\begin{tabular}{llllll}
\hline Ingredients (mg) & F1 & F2 & F3 & F4 & F5 \\
\hline Atorvastatin Calcium & 20 & 20 & 20 & 20 & 20 \\
Carbomer-974 & 75 & 100 & 100 & 75 & 75 \\
Hypromellose-15000cps & 75 & 75 & 100 & 100 & 75 \\
Starch & 150 & 125 & 100 & 100 & 100 \\
Sodium lauryl sulphate & - & - & - & 25 & 50 \\
Magnesium stearate & 5 & 5 & 5 & 5 & 5 \\
Total & 325 & 325 & 325 & 325 & 325 \\
\hline
\end{tabular}

\subsection{Evaluation of physical characterization}

The formulated tablets were evaluated for weight variation, thickness, hardness and diameter with regards to their British Pharmacopeia specification [10]. Compactibility assessment of all the prepared formulations were assessed by determining the force required for diametral breaking of the compacts using hardness tester (Electrolab EH$01 \mathrm{P})$. The tensile strength, $\sigma_{\mathrm{x}}$, is determined by the following equation [8].

$$
\sigma_{\mathrm{x}}=2 \mathrm{x} / \pi \mathrm{dt}
$$

Where, $x$ is hardness (kgf), $d$ is diameter $(\mathrm{mm})$ and $t$ is thickness (mm).

\subsection{Swelling studies}

Swelling property of tablet indicates the incorporation of liquid into the excipients that results in an increase in weight and volume. Thus, weight and volume are the parameters that can be used for measuring the extent of swelling. To measure swelling index, each tablet's diameter from all formulations were determined and then placed in a beaker containing $5 \mathrm{~mL}$ of phosphate buffer ( $\mathrm{pH} \mathrm{6.8)} \mathrm{solution} \mathrm{at} \mathrm{room} \mathrm{temperature.} \mathrm{After} 5 \mathrm{hr}$, the tablet was removed from the beaker and the diameter was determined. It is expressed as percentage, and was then calculated using the following equation:

$$
\mathrm{SI}=\left[\left(\mathrm{W}_{\mathrm{d}}-\mathrm{W}_{0}\right) / \mathrm{W}_{\mathrm{o}}\right] \times 100
$$

Where, $S I$ is swelling index, $W_{d}$ is diameter of tablet after immersion and $W_{o}$ is diameter of tablet before immersion. 


\subsection{Determination of drug content}

The drug content of the formulated products of each formulation was estimated by using UV spectrophotometer at $289 \mathrm{~nm}$ using suitable dilution.

\subsection{Dissolution (in-vitro drug release) studies}

Atorvastatin Calcium tablets in-vitro release studies was carried out in USP dissolution test apparatus II (paddle) with $50 \mathrm{RPM}$ rotation speed, using $900 \mathrm{~mL}$ of dissolution medium maintained at $37^{\circ} \mathrm{C} \pm 0.5^{\circ} \mathrm{C}$. Triplicate basket system was considered for dissolution studies using $900 \mathrm{~mL}$ of phosphate buffer (pH 6.8) as a dissolution medium. Test sample $(5 \mathrm{~mL})$ was withdrawn at particular time interval and replaced with fresh dissolution medium maintained at the same temperature and the concentration of dissolved drug was determined using UV spectrophotometer (Shimadzu) at $289 \mathrm{~nm}$.

\subsection{Kinetic evaluation of drug release data}

The release data from various batches of tablets obtained were subjected to release kinetic study by fitting into various postulated kinetic models. The drug release data from Atorvastatin Calcium tablets were analyzed by treating to zero-order [11] (cumulative amount of drug release versus time) and Higuchi [12] (cumulative percentage of drug release versus square root of time) equation models.

\section{Results and Discussion}

\subsection{Physical characterization of tablets}

The prepared tablets were preliminary analyzed for the evaluation of physical characteristics such as hardness, thickness, diameter and compactibility. The results obtained are summarized in Table 2. The hardness of tablets was found to be uniform within the range of $13.5-17 \mathrm{~kg} / \mathrm{ft}$. All the batches showed uniform thickness and diameter.

The values of tensile strength of the compacts are also presented in Table 2. The tensile strengths of all the formulations were $0.34-0.42$. This results indicate that Hypromellose-15000 cps and Carbomer-974 can be used as a polymer to solve the problems where the tablets showed insufficient tensile strength.

Table 2. Physical characteristics of Atorvastatin Calcium sustained release tablets.

\begin{tabular}{lllllll}
\hline \multirow{2}{*}{ Formulations } & \multicolumn{6}{c}{ Parameters } \\
\cline { 2 - 7 } & $\begin{array}{l}\text { Hardness } \\
(\mathrm{Kg} / \mathrm{ft})\end{array}$ & $\begin{array}{l}\text { Thickness } \\
(\mathrm{mm})\end{array}$ & $\begin{array}{l}\text { Diameter } \\
(\mathrm{mm})\end{array}$ & $\begin{array}{l}\text { Tensile } \\
\text { strength }\left(\sigma_{\mathrm{x}}\right)\end{array}$ & $\begin{array}{l}\text { Swelling } \\
\text { index }(\%)\end{array}$ & $\begin{array}{l}\text { Drug content } \\
(\%)\end{array}$ \\
\hline F1 & 15.06 & 2 & 12 & 0.399 & 50 & 97 \\
F2 & 16.04 & 2.2 & 12 & 0.387 & 41.66 & 80 \\
F3 & 17.04 & 2.15 & 12 & 0.421 & 58.33 & 95 \\
F4 & 16.36 & 2.25 & 12 & 0.386 & 66.67 & 81 \\
F5 & 13.57 & 2.1 & 12 & 0.342 & 45.83 & 97 \\
\hline
\end{tabular}




\subsection{Swelling index and drug content assessment}

The drug release profile may be dependent upon the swelling index of the prepared formulations. Swelling property of formulations, the ability to uptake liquid by the tablets, was determined for all the batches. The percentage of swelling of the formulations was determined at the end of $5 \mathrm{~h}$. Variation in the percent swelling was found with the variation of the concentration of polymers. The results of swelling index of formulations are presented in Table 2. The swelling index rate for all the formulations are: F4 > F3 > F1 > F5 > F2. From the results obtained, it showed that both the Hypromellose-15000 cps and Carbomer-974 increases the swelling property of the tablets but Hypromellose-15000 cps uptake more water than Carbomer-974. So, the floating ability of the prepared formulations depends on the polymer composition of the formulations.

The drug content of all the tablet formulations was analyzed. The results of percent drug content of various formulations are also depicted in Table 2. The percent drug content of all the formulations represented good uniformity and ranges from $80 \%$ to $97 \%$.

\subsection{In-vitro dissolution studies}

The in-vitro dissolution study of drug release was conducted in the USP paddle type apparatus using pH 6.8 phosphate buffer. The drug release profile over a period of $8 \mathrm{~h}$ was continuously monitored as the study aimed to develop a sustained release preparation with uplifted bioavailability. The result obtained from the dissolution study is illustrated in Table 3. The cumulative percent drug release from F1, F2, F3, F4 and F5 formulations were $52 \%, 48.2 \%, 51.95 \%, 58.5 \%$ and $35.5 \%$ respectively, within $8 \mathrm{~h}$ demonstrating the sustained release pattern. The formulation F4 showed the highest release of $58.5 \%$ up to 8 $\mathrm{hr}$ having increased amount of Hypromellose-15000 cps. Formulation F1 (150 mg starch) and F3 (100mg Carbomer-974, 100mg Hypromellose-15000 cps) also showed a good sustained release of $52 \%$ and $51.95 \%$ respectively after $8 \mathrm{~h}$ while Formulation F2 (48.2\%) and F5 $(35.5 \%)$ showed a comparatively more release retarding effect. The obtained data indicates that release of drug depends on the concentration of polymers and varying the concentration of polymer, Carbomer-974 and Hypromellose-15000 cps, the release retarding pattern of sustained release Atorvastatin Calcium tablets can be modulated.

Table 3. Percentage of drug release of Atorvastatin Calcium sustained release tablets of various formulations.

\begin{tabular}{|c|c|c|c|c|c|}
\hline \multirow{2}{*}{ Time (h) } & \multicolumn{5}{|c|}{$\%$ Drug release } \\
\hline & $\mathrm{F} 1$ & $\mathrm{~F} 2$ & F3 & $\mathrm{F} 4$ & F5 \\
\hline 0 & 0 & 0 & 0 & 0 & 0 \\
\hline 0.5 & 17.05 & 8.65 & 4.85 & 9.6 & 4.85 \\
\hline 1 & 19.95 & 10.55 & 8.6 & 11 & 16.15 \\
\hline 2 & 21.85 & 17.1 & 15.2 & 15.25 & 18.1 \\
\hline 3 & 25.65 & 19.05 & 19.95 & 18.1 & 20.95 \\
\hline 4 & 28.45 & 20.95 & 20.95 & 20.95 & 25.65 \\
\hline 5 & 30.4 & 23.75 & 25.65 & 22.8 & 27.50 \\
\hline
\end{tabular}




\begin{tabular}{llllll}
\hline Time $(\mathrm{h})$ & \multicolumn{2}{l}{$\%$ Drug release } & & \\
\cline { 2 - 6 } & F1 & F2 & F3 & F4 & F5 \\
\hline 6 & 38.8 & 29.4 & 29.4 & 24.7 & 30.35 \\
7 & 43.55 & 35.05 & 32.25 & 35 & 32.25 \\
8 & 52 & 48.2 & 51.95 & 58.5 & 35.5 \\
\hline
\end{tabular}

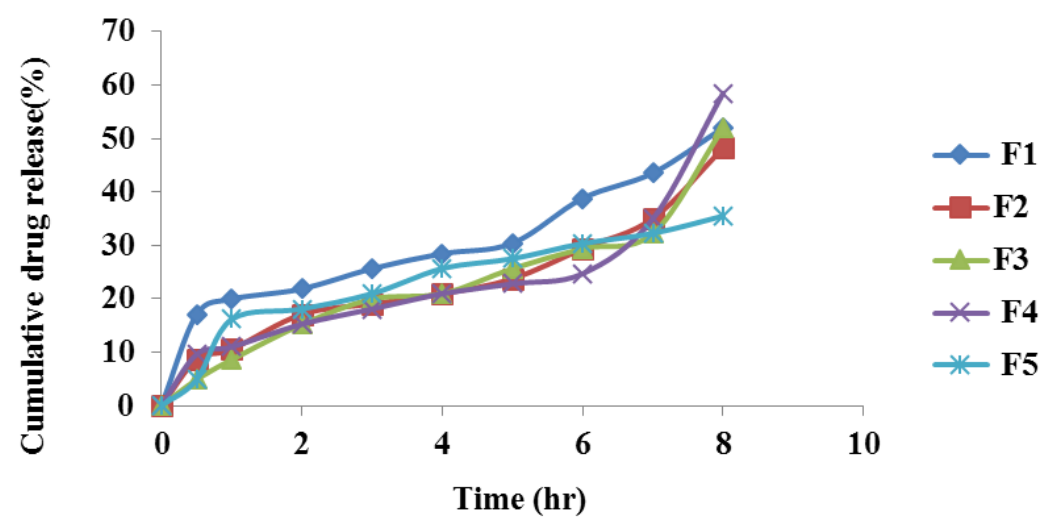

Fig. 1. Zero order release pattern of formulated Atorvastatin Calcium sustained release tablet.

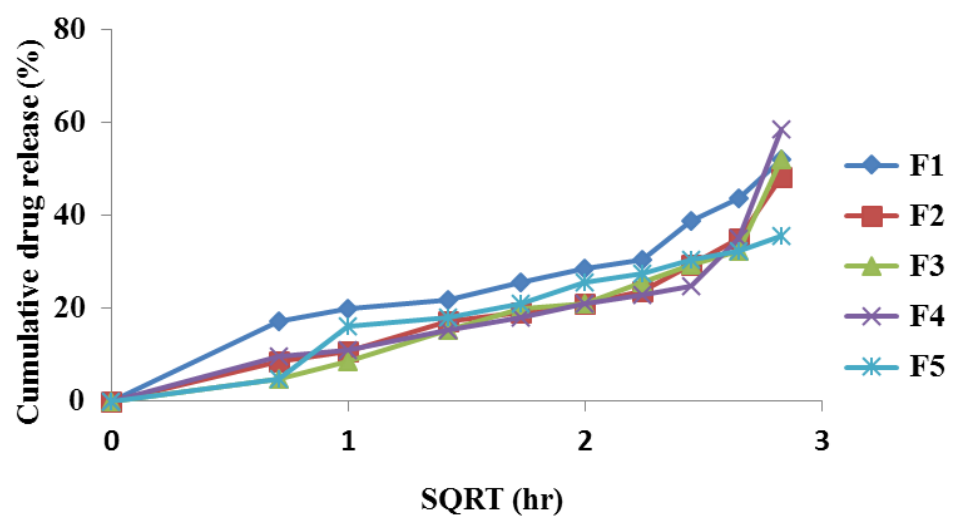

Fig. 2. Higuchi release pattern for formulated Atorvastatin Calcium sustained release tablet.

\subsection{Kinetic evaluation of drug release studies}

In order to investigate the release mechanism of the various sustained release formulations of Atorvastatin Calcium, the release data of the formulations were applied to various drugrelease kinetic models. The release rates were evaluated by Zero order and Higuchi model, which have been suggested to describe the drug release kinetics from various formulations. Zero order plot and Higuchi plot release pattern of the five prepared formulations are depicted in Figs. 1 and 2 respectively. The interpretation of data was 
based on the values of the resulting regression co-efficient as depicted in Table 4. The results showed that in F1 and F5 formulations where both Carbomer-974 and Hypromellose- $15000 \mathrm{cps}$ were used $75 \mathrm{mg}$ for each formulation, best fitted in the Higuchi model having $\mathrm{R}^{2}$ value 0.929 and 0.974 respectively, indicating drug release through the diffusion process. On the contrary, formulations F2, F3 and F4, where composition of both Carbomer-974 and Hypromellose-15000 cps concentration was varied, showed the best fitting in the Zero order model compared to Higuchi model (Table 4), having $\mathrm{R}^{2}$ value $0.936,0.927$ and 0.834 respectively, indicating prolonged drug release over time. These formulations are not fitted in Higuchi model as these showed high swelling property compared to other and Higuchi model cannot be applicable in release pattern of drug where swelling is predominant.

Table 4. Release kinetics of Atorvastatine Calcium sustained release tablet.

\begin{tabular}{lll}
\hline Formulations & Zero order $\left(\mathrm{R}^{2}\right)$ & Higuchi $\left(\mathrm{R}^{2}\right)$ \\
\hline F1 & 0.908 & 0.929 \\
F2 & 0.936 & 0.890 \\
F3 & 0.927 & 0.867 \\
F4 & 0.834 & 0.749 \\
F5 & 0.892 & 0.974 \\
\hline
\end{tabular}

\section{Conclusion}

On the basis of the present study, it can be exemplified that Atorvastatin Calcium sustained release tablets can be formulated using Carbomer-974 and Hypromellose-15000 cps polymer along with other excipients. The study depicts that the proposed formulations can be used to prepare tablets using direct compression method and the addition of Carbomer-974 and Hypromellose-15000 cps as rate controlling polymer represented good sustained drug release rate and improved physical properties. The drug content, release pattern and swelling index property of the formulated preparations are concentration dependent of the polymers. So, varying the concentration and composition of polymers the sustained release pattern of the formulations can be modulated. This study will provide sufficient lend mark for further research studies of this drug delivery system for the benefit of mankind.

\section{References}

1. H. Yousaf, M. Jamshaid, I. Bashir, and Y. Mehmood, Pharm Methods 8(1), 183 (2017). doi: $10.5530 / \mathrm{phm} .2017 .8 .8$

2. L. L. Augsburger and M. J. Zellhofer, Encyclo. Pharm. Technol. 3641 (2006).

3. A. Corsini, S. Bellosta, R. Baetta, R. Fumagalli, R. Paoletti, and F. Bernini, Pharmacol. Ther. 84 (3), 413 (1999). http://dx.doi.org/10.1016/S0163-7258(99)00045-5

4. M. Z. Rahman, S. K. Ahamed, S. Banik, and M. S. Hossain, Bangladesh Pharm. J. 16(2), 177 (2013). https://doi.org/10.4135/9781452276151.n33

5. S. K. Ahamed, S. Banik, and M. S. Hossain, Int. J. Pharm. Sci. Res. 4(3), 1140 (2013). https://doi.org/10.13040/IJPSR.0975-8232.4(3).1140-47

6. L. Kukati, K. Chittimalli, and N. B. Shaik, J. Sci. Res. 6(3), 563 (2014). 
https://doi.org/10.3329/jsr.v6i3.18339

7. H. Shato, Y. Miyagawa, T. Okabe, M. Miyajima, and H. Sunada, J. Pharm. Sci. 86(8), 929 (1997). https://doi.org/10.1021/js960221w

8. M. S. Hossain, S. Banik, and M. S. Islam, Ind. J. Pharm. Edu. Res. 46 (2), 136 (2012).

9. M. M. K. Bhuiyan, S. Banik, M. A. Hasan, M. S. Reza, and M. S. Hossain, Marmara Pharm. J. 21(2), 59 (2017). https://doi.org/10.12991/marupj.259882

10. British Pharmacopoeia. Her Majesty's Stationary Office (London, England, 2000).

11. J. Cooper and C. Gunn, Tutorial pharmacy, $6^{\text {th }}$ ed. (CBS publishers and Distributors, New Delhi, 1986) pp. 225.

12. T. Higuchi, J. Pharm. Sci. 52 (12), 1145 (1963). https://doi.org/10.1002/jps.2600521210 